TRANSACTIONS OF THE

AMERICAN MATHEMATICAL SOCIETY

Volume 358, Number 8, Pages 3673-3695

S 0002-9947(06)03995-X

Article electronically published on March 28, 2006

\title{
ON THE ESHELBY-KOSTROV PROPERTY FOR THE WAVE EQUATION IN THE PLANE
}

\author{
M. A. HERRERO, G. E. OLEAGA, AND J. J. L. VELÁZQUEZ
}

\begin{abstract}
This work deals with the linear wave equation considered in the whole plane $\mathbb{R}^{2}$ except for a rectilinear moving slit, represented by a curve $\Gamma(t)=\left\{\left(x_{1}, 0\right):-\infty<x_{1}<\lambda(t)\right\}$ with $t \geq 0$. Along $\Gamma(t)$, either homogeneous Dirichlet or Neumann boundary conditions are imposed. We discuss existence and uniqueness for these problems, and derive explicit representation formulae for solutions. The latter have a simple geometrical interpretation, and in particular allow us to derive precise asymptotic expansions for solutions near the tip of the curve. In the Neumann case, we thus recover a classical result in fracture dynamics, namely the form of the stress intensity factor in crack propagation under antiplane shear conditions.
\end{abstract}

\section{INTRODUCTION}

The Eshelby-Kostrov (EK) property is an important result in the theory of dynamic fracture propagation. In mathematical terms, it consists in a remarkable feature of the classical wave equation, that can be described as follows. Set $\mathbf{x}=$ $\left(x_{1}, x_{2}\right)$, and assume that $u(\mathbf{x}, t)$ solves the linear wave equation in a slit domain in the plane

$$
u_{t t}-\Delta u=0 \quad \text { in } \mathbb{R}^{2} \backslash \Gamma(t), t>0
$$

where

$$
\Gamma(t)=\left\{\left(x_{1}, 0\right):-\infty<x_{1}<\lambda(t)\right\} .
$$

The slit curve $\lambda(t)$ (which will often be termed as a moving crack) is an increasing smooth function moving at subsonical speed:

$$
0 \leq \dot{\lambda}(t)<1, t>0 \text {. }
$$

Without loss of generality we will assume that $\lambda(0)=0$. Along $\Gamma(t), u$ satisfies a homogeneous Neumann boundary condition

$$
\frac{\partial u}{\partial n}=0 \text { in } \Gamma(t), t>0 \text {, }
$$

and $u, u_{t}$ take suitable initial values $u_{0}, v_{0}$ at $t=0$. Under some assumptions to be made precise later, the function $u(\mathbf{x}, t)$, satisfying the previous requirements, can be expanded asymptotically near the tip of $\Gamma(t)$ in the form

$$
u(\mathbf{x}, t) \sim K(t)|\mathbf{x}-\lambda(t)|^{1 / 2} B(\theta, \dot{\lambda}(t)) \quad \text { as } \quad \mathbf{x} \rightarrow \lambda(t),
$$

Received by the editors September 15, 2003 and, in revised form, September 12, 2004

2000 Mathematics Subject Classification. Primary 74R05, 74B05, 74G70.

(C)2006 American Mathematical Society 
where $B(\theta, \dot{\lambda}(t))$ is a suitable angular factor depending on the angle $\theta$ subtended by the vector $\mathbf{x}-\lambda(t)$ and the $x_{1}$-axis. In principle, one would expect $K(t)$ to depend on the whole evolution of the function $\lambda(t)$, i.e.,

$$
K(t)=K\left(\{\lambda(s), 0 \leq s \leq t\}, u_{0}, v_{0}\right) .
$$

However, it turns out that this is not the case, but that on the contrary, $K(t)$ depends only on the local properties of $\lambda(t)$. More precisely, there exist functions $K_{1}, K_{2}$ such that

$$
K(t)=K_{1}\left(\lambda(t) ; u_{0}, v_{0}\right) K_{2}(\dot{\lambda}(t)) \equiv K_{1}(\lambda(t)) K_{2}(\dot{\lambda}(t)) .
$$

Condition (1.6) is the so-called EK property. It was initially derived, under suitable assumptions on the initial data, by Eshelby (cf. 2]) and Kostrov (cf. [10]). Eshelby's proof is only valid when $v_{0}=0$ (in the absence of body forces), and $u_{0}$ is a solution of the stationary version of (1.1), (1.4), with the slit curve remaining at rest, $\Gamma(t)=\Gamma(0)$ for all $t \geq 0$. Furthermore, Eshelby's argument requires sophisticated computations involving power series. On the other hand, Kostrov's approach, which is valid for a more general class of data, is based on deriving clever representation formulae for solutions that rely on the use of WienerHopf and integral equations techniques. A somewhat simpler argument was given by Freund (cf. [4]), where this property is derived by solving first the problem for the particular case $\dot{\lambda}(t)=$ constant, and then using these particular solutions to approximate $u(\mathbf{x}, t)$ in the region $x \sim \lambda(t)$ for arbitrary smooth functions $\lambda(t)$.

The relevance of the EK property stems from the fact that, in the case of planar fracture, it allows us to replace the full free boundary problem of crack propagation by a considerably simpler ordinary differential equation. Indeed, standard crack propagation criteria are often given in the form

$$
K(t)=K_{c},
$$

where $K_{c}$ is a material parameter (usually termed as the fracture toughness) of the media under consideration. In view of (1.6), (1.7) can then be reduced to solving a nonlinear ODE, namely

$$
K_{1}(\lambda(t)) K_{2}(\dot{\lambda}(t))=K_{c}
$$

cf. for instance [4. We remark in passing that determining the stress intensity factor function $K(t)$ in the course of the slit motion is a major problem in fracture mechanics; see for instance [1], [5], 6], 8], 11] and the references therein.

The goal of this paper consists in providing a comparatively simple derivation of the EK property. In our opinion this approach has a number of advantages. To begin with, it allows us to derive such property under less restrictive assumptions, and in more general situations: for instance, the case of homogeneous Dirichlet conditions instead of (1.4) can be readily handled. Furthermore, our method is conceptually simpler, allows for a natural geometric interpretation, and requires only elementary computations. It should be noted, however, that the full validity of the EK property seems to be restricted to the case of rectilinear slit motion. As a matter of fact, we have recently shown that when kinked paths in the plane are considered, that property does not hold in general (cf. [8]). In this paper, we will make use of classical methods based on complex variable theory (conformal mapping, Wiener-Hopf techniques, ...) which have been extensively employed to 
obtain representation formulae for particular fracture problems (cf. for instance [4, [14, [7, [12, [15]).

This article is organised as follows. In Section 2 we consider a particular choice of initial data, whose analysis we retain to be particularly instructive. The main result there is Theorem 2.1] a reformulation of Eshelby's results, with a simpler proof applicable to both Dirichlet and Neumann boundary conditions. In Section 3 we generalize the main idea to arbitrary initial data for both kinds of initial-boundary value problems. The corresponding results are summarized in Theorem 3.1 therein. We conclude that section with a geometric discussion of the representation formulae obtained. Finally, a number of auxiliary results are recalled in an Appendix at the end of the paper.

\section{The Eshelby Case: A particular Choice of initial data}

Let us denote by $C_{c}^{0, \alpha}\left(\mathbb{R}^{2}\right)$ the set of functions which are compactly supported in $\mathbb{R}^{2}$ and satisfy a Hölder condition with exponent $\alpha$, i.e., such that

$$
|f(\mathbf{x})-f(\mathbf{y})| \leq C|\mathbf{x}-\mathbf{y}|^{\alpha},
$$

for some $C>0$ and $0<\alpha<1$. Suppose that $u$ satisfies the following equation:

$$
\square u \equiv \frac{\partial^{2} u}{\partial t^{2}}-\Delta u=f(\mathbf{x}), \quad \mathbf{x} \in \mathbb{R}^{2} \backslash \Gamma(t), t>0,
$$

where $f \in C_{c}^{0, \alpha}\left(\mathbb{R}^{2}\right)$ and $\Gamma(t)$ is as in (1.2). In this section we will solve (2.1) for a particular class of initial data and with homogeneous Dirichlet or Neumann boundary conditions on $\Gamma(t)$. More precisely, let us define

$$
\Gamma_{a}:=\left\{\mathbf{x}=\left(x_{1}, 0\right):-\infty<x_{1}<a\right\}, a \geq 0 .
$$

Consider the following problem:

$$
\begin{gathered}
-\Delta \phi(\mathbf{x} ; a)=f(\mathbf{x}) \quad \text { for } \mathbf{x} \in \mathbb{R}^{2} \backslash \Gamma_{a}, \\
\phi(\mathbf{x} ; a)=0 \quad \text { for } \mathbf{x} \in \Gamma_{a},
\end{gathered}
$$

or respectively,

$$
\frac{\partial \phi}{\partial n}(\mathbf{x} ; a)=0 \quad \text { for } \mathbf{x} \in \Gamma_{a}
$$

We shall see that (2.3), (2.4) (respectively (2.3), (2.5)) has a unique solution $\phi_{D}$ (respectively a solution unique up to a constant, $\phi_{N}$ ) in a suitable class of functions. More precisely, there holds:

Lemma 2.1. Let $f$ be as before. Then for each fixed $a \geq 0$, problem (2.3), (2.4) has a unique solution $\phi(\mathbf{x} ; a) \equiv \phi_{D}(\mathbf{x} ; a)$ satisfying

$$
\phi(\mathbf{x} ; a) \rightarrow 0 \quad \text { as } \quad|\mathbf{x}| \rightarrow \infty
$$

and for any $R>a$, there exists $C=C(R)<\infty$ such that:

$$
\int_{\{|\mathbf{x}|<R\} \backslash \Gamma_{a}}|\nabla \phi|^{2} d x_{1} d x_{2}<C(R) .
$$

On the other hand, problem (2.3), (2.5) has a solution $\phi(\mathbf{x} ; a) \equiv \phi_{N}(\mathbf{x} ; a)$ which is unique up to the addition of an arbitrary constant and is such that (2.7) holds, and

$$
\phi(\mathbf{x} ; a)=O(\log (|x|)) \quad \text { as } \quad \mathbf{x} \rightarrow \infty .
$$


Proof. It is rather standard and will be sketched here for convenience of the reader. To begin with, we subtract the contribution due to the source $f(x)$, that can be easily computed by means of the fundamental solution for the Laplace equation:

$$
\phi_{f}(\mathbf{x}) \equiv \frac{1}{2 \pi} \int_{\mathbb{R}^{2}} \log \left(\frac{1}{|\mathbf{x}-\mathbf{y}|}\right) f(\mathbf{y}) d \mathbf{y} .
$$

Therefore the function $\psi=\phi-\phi_{f}$ is harmonic in $\mathbb{R}^{2} \backslash \Gamma_{a}$ and satisfies the boundary conditions

$$
\psi=-\phi_{f} \quad \text { for } \mathbf{x} \in \Gamma_{a},
$$

in the case where (2.4) holds, and

$$
\frac{\partial \psi}{\partial n}=-\frac{\partial \phi_{f}}{\partial n} \quad \text { for } \mathbf{x} \in \Gamma_{a},
$$

when (2.5) is satisfied. In either case, the function $\psi$ can be readily computed using the conformal mapping $z \rightarrow \xi=\sqrt{z}$, that transforms the slit domain in the halfplane $\operatorname{Re}(z)>0$, combined with the classical representation formula for harmonic functions in a half-plane in terms of their boundary data. It then follows that

$$
\psi(\mathbf{x}) \equiv \psi(z)=-\frac{1}{\pi} \int_{0}^{\infty} \phi_{f}(-\lambda) \operatorname{Re}\left(\frac{\sqrt{z}}{z+\lambda}\right) \frac{d \lambda}{\sqrt{\lambda}},
$$

in the case of Dirichlet boundary data and

$$
\psi(\mathbf{x}) \equiv \psi(z)=-\frac{1}{\pi} \int_{0}^{\infty} \frac{\partial \phi_{f}(-\lambda)}{\partial y} \log \left(\frac{|i \sqrt{\lambda}-\sqrt{z}|}{|i \sqrt{\lambda}+\sqrt{z}|}\right) d \lambda,
$$

for Neumann boundary values. In the two formulae above we assume that $z=$ $x_{1}+i x_{2}$.

Making use of the asymptotic behaviour of $\phi_{f}$ (which is readily obtained from (2.9) and the assumptions made on $f$ ) it is easily seen that the integrals in (2.10) and (2.11) converge. Therefore $\phi_{D}(\mathbf{x} ; a)=\psi(\mathbf{x})+\phi_{f}(\mathbf{x})$ provides a solution of (2.3), (2.4) and (2.7). On the other hand, from (2.9) and (2.10) it follows that $\phi_{D}(\mathbf{x} ; a)=O(\log (|\mathbf{x}|))$ as $|\mathbf{x}| \rightarrow \infty$. Using the conformal mapping $z \rightarrow \frac{1}{\sqrt{z}}=w$ that brings the point at infinity to the origin, and setting $\mathbf{w}=\left(w_{1}, w_{2}\right)$ where $w=w_{1}+i w_{2}$, we obtain a function $\bar{\phi}_{D}(\mathbf{w} ; a)=\phi_{D}(\mathbf{x} ; a)$, which is harmonic in a half-plane, and satisfies the bound $\bar{\phi}_{D}(\mathbf{w} ; a)=O\left(\log \left(\frac{1}{|\mathbf{w}|}\right)\right)$ as $|\mathbf{w}| \rightarrow 0$. We then take advantage of supersolutions of the form $\hat{\phi}_{D}(\mathbf{w} ; a)=C+\frac{\varepsilon}{|\mathbf{w}|^{\alpha}} \cos (\alpha \theta)$ with $\alpha>0$ small and $\varepsilon \rightarrow 0$, to deduce that $\bar{\phi}_{D}(\mathbf{w} ; a)$ is bounded near the origin and therefore, by standard regularity theory, it follows that $\phi_{D}$ satisfies (2.6). In the case of Neumann conditions, using in (2.11) the change of variables $\lambda=|z| u$ as well as the fact that $\frac{\partial \phi_{f}(-\lambda)}{\partial y}=O\left(\frac{1}{\lambda}\right)$ as $\lambda \rightarrow \infty$, we obtain that $\psi(z)=O(1)$ as $|z| \rightarrow \infty$, whence (2.8) follows.

The uniqueness result can also be proved by means of conformal mapping. Indeed, first consider the case of Dirichlet conditions. After transforming the domain $\mathbb{R}^{2} \backslash \Gamma_{a}$ in the unit disk, we obtain that the difference of two solutions should be a bounded harmonic function in a circle with zero Dirichlet boundary conditions. In the Neumann case, arguing as before, we transform the domain $\mathbb{R}^{2} \backslash \Gamma_{a}$ in a half-plane $\operatorname{Re}(w)>0$ and the point $z=\infty$ in the origin, where the resulting function would be bounded by a term of the form $o\left(\log \left(\frac{1}{|\mathbf{w}|}\right)\right)$. Indeed, conformal 
mapping plus integration by parts yield that for arbitrary solutions of (2.3), (2.7), (2.5), (2.8),

$$
\phi_{N}(\mathbf{x} ; a) \sim \frac{\log (|\mathbf{x}|)}{2 \pi} \int_{\mathbb{R}^{2}} f(y) d y \quad \text { as }|x| \rightarrow \infty,
$$

whence the difference $W$ of two solutions of this problem satisfies the inequality $W=o(\log (|\mathbf{x}|))$ as $|\mathbf{x}| \rightarrow \infty$. The transformed solution in the half-plane $\operatorname{Re}(w)>0$ can then be extended by symmetry to a harmonic function in $\mathbb{R}^{2}$ that is a constant, as can be seen by means of a Liouville's theorem-type argument. This concludes the proof.

In the sequel, we will denote by $\left(P_{D}\right)$ the problem consisting of (2.1) with homogeneous Dirichlet boundary condition

$$
u=0 \text { in } \Gamma(t),
$$

as well as the initial data

$$
\begin{aligned}
u(\mathbf{x}, 0) & =\phi_{D}(\mathbf{x} ; 0), \quad \mathbf{x} \in \mathbb{R}^{2} \backslash \Gamma(0), \\
u_{t}(\mathbf{x}, 0) & =0, \quad \mathbf{x} \in \mathbb{R}^{2} \backslash \Gamma(0) .
\end{aligned}
$$

On the other hand, we will denote by $\left(P_{N}\right)$ the problem consisting of equation (2.1) with boundary condition

$$
\frac{\partial u}{\partial n}=0 \text { in } \Gamma(t),
$$

and initial data

$$
\begin{aligned}
u(\mathbf{x}, 0) & =\phi_{N}(\mathbf{x} ; 0), \quad \mathbf{x} \in \mathbb{R}^{2} \backslash \Gamma(0), \\
u_{t}(\mathbf{x}, 0) & =0, \quad \mathbf{x} \in \mathbb{R}^{2} \backslash \Gamma(0) .
\end{aligned}
$$

Notice that in problems $\left(P_{D}\right),\left(P_{N}\right)$ we assume that the initial data are stationary solutions of (2.1) for a fixed curve, $\Gamma(t)=\Gamma(0)$ for all $t>0$.

The following result provides a representation formula for the solutions of $\left(P_{D}\right)$ and $\left(P_{N}\right)$ in terms of the family of functions $\phi_{D}, \phi_{N}$ for general subsonic functions $\lambda(t)$. Following Eshelby [2, we introduce an auxiliary function $\tau(\mathbf{x}, t)$ for $|\mathbf{x}| \leq t$ that will play a key role in the sequel by means of the formula

$$
|\mathbf{x}-\lambda(\tau(\mathbf{x}, t))|=t-\tau(\mathbf{x}, t), \quad 0 \leq \tau \leq t .
$$

Note that the quantity $\tau(\mathbf{x}, t)$ given in (2.19) is well defined. Indeed, for any $t>$ 0 , for each value of $0 \leq \tau \leq t$ we may define a circumference $C_{\tau}=\{\mathbf{x}:|\mathbf{x}-\lambda(\tau)|=$ $t-\tau\}$. We have that $C_{t}=\lambda(t)$, and $C_{0}=\{\mathbf{x}:|\mathbf{x}|=t\}$. Note that for $\tau_{1}<\tau_{2}$ and $\dot{\lambda}(t) \leq 1, C_{\tau_{2}}$ is strictly contained in the circle enclosed by $C_{\tau_{1}}$. Therefore, the union of the sets $C_{\tau}$ for $0 \leq \tau \leq t$ covers the whole disk $\{\mathbf{x}:|\mathbf{x}| \leq t\}$, and then for each point $\mathbf{x}$ there is a unique $\tau(\mathbf{x}, t)$ such that $\mathbf{x} \in C_{\tau(\mathbf{x}, t)}$.

We now have:

Theorem 2.1. For any function $\lambda(\cdot) \in C^{2}([0, \infty])$ satisfying (1.3) as well as $\lambda(0)=0$, and for any function $f(\cdot) \in C_{c}^{0, \alpha}\left(\mathbb{R}^{2}\right)(0<\alpha<1)$ there exists a solution $u(x, t)$ of problem $\left(P_{D}\right)$, with initial data

$$
u(\mathbf{x}, 0)=\phi_{D}(\mathbf{x} ; 0), \quad u_{t}(\mathbf{x}, 0)=0,
$$




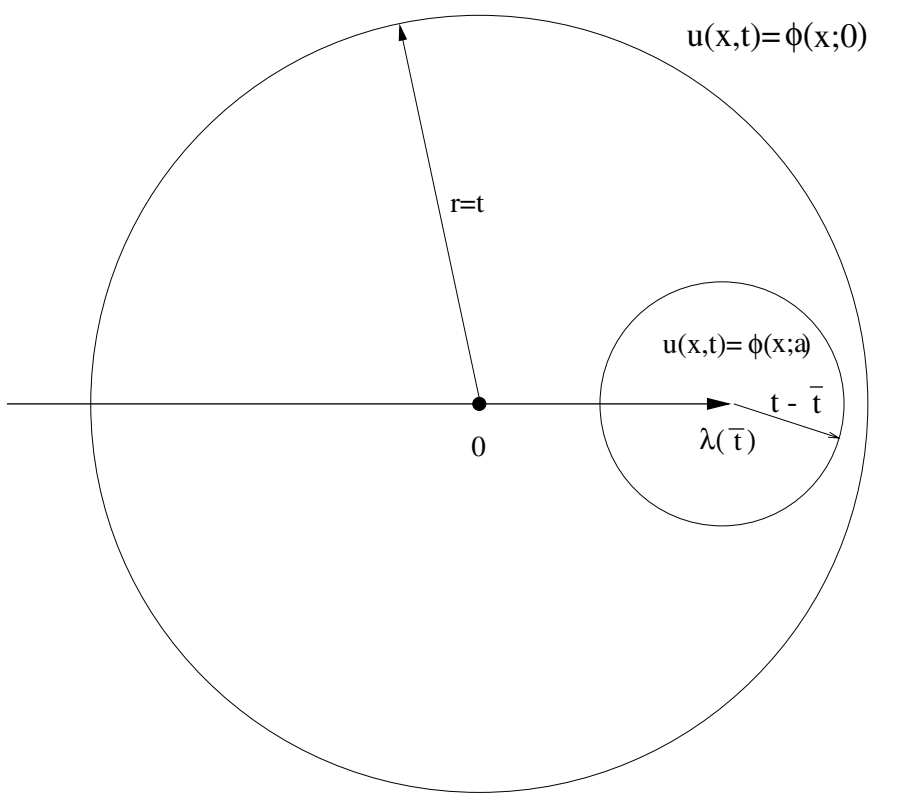

Figure 1. The structure of solutions of the Eshelby problem: for $a \geq 0, \phi(\mathbf{x}, a)$ stands for $\phi_{D}(\mathbf{x}, a)$ or $\phi_{N}(\mathbf{x}, a)$.

where the function $\phi_{D}$ is as in (2.3), (2.4), (2.6) and (2.7). The corresponding function $u$ is given by

$$
u(\mathbf{x}, t)=\phi_{D}(\mathbf{x} ; \lambda(\tau(\mathbf{x}, t)))
$$

where $\tau(\mathbf{x}, t)$ is as in (2.19). In the case of problem $\left(P_{N}\right)$, for each $a \geq 0$ it is possible to select $\phi_{N}$, among the monoparametric family of solutions of (2.3), (2.5), (2.7), (2.8) in such a way that (2.20) (with $\phi_{D}$ replaced by $\phi_{N}$ there) provides a solution of $\left(P_{N}\right)$ with initial conditions

$$
u(\mathbf{x}, 0)=\phi_{N}(\mathbf{x} ; 0), \quad u_{t}(\mathbf{x}, 0)=0 .
$$

Theorem 2.1 has a simple geometrical interpretation as follows. If the slit curve $\lambda(t)$ arrests at some time $t=\bar{t}, u(\mathbf{x}, t)=\phi_{D}(\mathbf{x}, \lambda(\bar{t}))\left(\operatorname{resp} . u(\mathbf{x}, t)=\phi_{N}(\mathbf{x}, \lambda(\bar{t}))\right)$ at the interior of the cone $\left(x_{1}-\lambda(\bar{t})\right)^{2}+x_{2}^{2}=(t-\bar{t})^{2}$. On the other hand, for times $t>\bar{t}$, no information about the manner of the motion reaches to the region $\left(x_{1}-\lambda(0)\right)^{2}+x_{2}^{2}>t^{2}$, so that $u(\mathbf{x}, t)=\phi_{D}(\mathbf{x}, 0)\left(u(\mathbf{x}, t)=\phi_{N}(\mathbf{x}, 0)\right)$ there; see Figure 1.

The solutions described in Theorem 2.1 can be shown to be unique in a suitable functional class. However, a discussion on uniqueness will be postponed to Section 3 below. Indeed, our main goal herein consists in showing that the model problems $\left(P_{D}\right),\left(P_{N}\right)$ admit explicit solutions as that given by (2.20), and whose geometrical meaning has just been remarked upon. 
To proceed further, we show:

Lemma 2.2. Suppose that $\phi_{D}(\mathbf{x} ; a)$ is as in Lemma 2.1. Then $\phi_{D}(\mathbf{x} ; a)$ is continuously differentiable with respect to $a$, and the function $\frac{\partial \phi_{D}}{\partial a}$ is such that

$$
\frac{\partial \phi_{D}}{\partial a}(\mathbf{x} ; \lambda)+2 \nabla\left(\frac{\partial \phi_{D}}{\partial a}\right)(\mathbf{x} ; \lambda) \cdot(\mathbf{x}-\lambda)=0
$$

for any $\lambda \geq 0$. On the other hand, it is possible to select a family of functions $\phi_{N}(\mathbf{x} ; a)$ in such a way that $\phi_{N}(\mathbf{x} ; a)$ is continuously differentiable with respect to $a$, and $\frac{\partial \phi_{N}}{\partial a}$ satisfies (2.21).

Proof. In what follows we identify the complex variable $z=x_{1}+i x_{2}$ with $\mathbf{x} \in \mathbb{R}^{2}$. After transforming the domain $\mathbb{R}^{2} \backslash \Gamma_{a}$ in the half-plane $\{\operatorname{Re}(w)>0\}$ by means of the conformal mapping $z \rightarrow \sqrt{z-a}=w$, we obtain that $\tilde{\phi}(w)=\phi(z)$ solves $\Delta_{w} \tilde{\phi}=4|w|^{2} f\left(a+w^{2}\right)$. Classical regularity theory for the Laplace equation implies that $\tilde{\phi}$ is twice differentiable at $w=0$, with uniform bounds for the derivatives with respect to $a$. Returning to the original variable $z$, we readily see that $\phi$ behaves asymptotically near $z=a$ as follows:

$$
\begin{aligned}
\phi_{D}(z ; a)= & K_{1}(a) \operatorname{Re}(\sqrt{z-a})+K_{2}(a) \operatorname{Im}(z-a) \\
& +K_{3}(a) \operatorname{Re}\left((z-a)^{3 / 2}\right)+O\left((z-a)^{2}\right), \\
\phi_{N}(z ; a)= & C_{0}(a)+K_{1}(a) \operatorname{Im}(\sqrt{z-a})+K_{2}(a) \operatorname{Re}(z-a) \\
& +K_{3}(a) \operatorname{Im}\left((z-a)^{3 / 2}\right)+O\left((z-a)^{2}\right),
\end{aligned}
$$

where $C_{0}(a), K_{i}(a)(1 \leq i \leq 3)$ are suitable real constants depending on $a$ and $f$, although this last dependence will not be made explicit in the following.

Let us first discuss $\frac{\partial \phi_{D}}{\partial a}$. To this end, we consider the incremental ratios $\psi_{h}(\mathbf{x} ; a)$ $=\frac{\phi_{D}(\mathbf{x} ; a+h)-\phi_{D}(\mathbf{x} ; a)}{h}$. Assume that $h>0$, since the case $h<0$ could be handled in a similar manner. Note that by definition of $\phi_{D}$ we have that $\psi_{h}$ is harmonic in $\mathbb{R}^{2} \backslash \Gamma_{a+h}$ and takes the boundary values

$$
\begin{aligned}
& \psi_{h}(\mathbf{x} ; a)=0 \text { in } \Gamma_{a}, \\
& \psi_{h}(\mathbf{x} ; a)=-\frac{1}{h} \phi_{D}(\mathbf{x} ; a) \text { in } \Gamma_{a+h} \backslash \Gamma_{a}, \\
& \psi_{h}(\mathbf{x} ; a) \rightarrow 0 \text { as }|\mathbf{x}| \rightarrow \infty
\end{aligned}
$$

Using the conformal mapping $z \rightarrow \sqrt{z-a-h}=w$ we obtain that $\tilde{\psi}_{h}(w)=$ $\psi_{h}(z)$ is harmonic in $\{\operatorname{Re}(w)>0\}$ (in the case $h<0$ this conformal map is to be replaced by $z \rightarrow \sqrt{z-a})$. The function $\tilde{\psi}_{h}$ vanishes on the line $\operatorname{Re}(w)=0$ when $|w| \geq \sqrt{h}$. On the other hand, using (2.22) a quick computation reveals that, in the segment $\operatorname{Re}(w)=0,|w|<\sqrt{h}$,

$$
\tilde{\psi}_{h}(w)=-\frac{K_{1}(a)}{h} \operatorname{Re}\left(\sqrt{h-w_{2}^{2}}\right)+O(1) \text { as } h \rightarrow 0,
$$

where $w=w_{1}+i w_{2}$. Using the explicit representation formula for a harmonic function in a half-plane in terms of its boundary values at $\operatorname{Re} w=0$, we then have that, when $|w| \geq \sqrt{h}$ and $h \rightarrow 0$,

$$
\tilde{\psi}_{h}(w)=-\frac{K_{1}(a)}{\pi h} \int_{|\eta|<\sqrt{h}} \frac{w_{1} \operatorname{Re}\left(\sqrt{h-\eta^{2}}\right)}{w_{1}^{2}+\left(w_{2}-\eta\right)^{2}} d \eta+O\left(\frac{h^{1 / 2} w_{1}}{w_{1}^{2}+w_{2}^{2}}\right) .
$$


Then, uniformly on compact sets of $\mathbb{R}^{2} \backslash \Gamma_{a}$, we have that

$$
\frac{\partial \phi_{D}}{\partial a}=\lim _{h \rightarrow 0}\left(\tilde{\psi}_{h}(w)\right)=-\frac{K_{1}(a)}{2} \operatorname{Re}\left(\frac{1}{\lim _{h \rightarrow 0} w}\right)=-\frac{K_{1}(a)}{2} \operatorname{Re}\left(\frac{1}{\sqrt{z-a}}\right) .
$$

On differentiating in (2.25), (2.21) follows.

The proof for $\frac{\partial \phi_{N}}{\partial a}$ is basically analogous, but some remarks are in order. Since $\phi_{N}$ is only defined up to the addition of an arbitrary constant, the value of $C_{0}$ in (2.23) can be chosen in an arbitrary manner, and we may prescribe $C_{0}(a) \equiv$ constant for all $a \geq 0$. One also has to replace the Poisson kernel appearing for instance in (2.24) by the corresponding kernel associated to Neumann boundary conditions. Instead of (2.25) we now have

$$
\frac{\partial \phi_{N}}{\partial a}(z ; a)=-\frac{K_{1}(a)}{2} \operatorname{Im} \frac{1}{\sqrt{z-a}} .
$$

Note that we have derived an explicit formula for $\frac{\partial \phi}{\partial a}$ in terms of $K_{1}(a)$ (cf. (2.25)). Integrating this equation we obtain

$$
\phi_{D}(x, a)=\phi_{D}(x, 0)-\int_{0}^{a} \frac{K_{1}(\eta)}{2} \operatorname{Re} \frac{1}{\sqrt{z-\eta}} d \eta \text {. }
$$

A similar expression for $\phi_{N}$ is readily obtained from (2.26), namely

$$
\phi_{N}(x, a)=\phi_{N}(x, 0)-\int_{0}^{a} \frac{K_{1}(\eta)}{2} \operatorname{Im} \frac{1}{\sqrt{z-\eta}} d \eta .
$$

We next prove:

Lemma 2.3. Under the same conditions of Lemma 2.2 , we have that $\phi_{D}(\mathbf{x} ; a)$ and $\phi_{N}(\mathbf{x} ; a)$ are twice differentiable with respect to a for $\mathbf{x} \in \mathbb{R}^{2} \backslash \Gamma_{a}$.

Proof. We only consider the problem with Dirichlet boundary conditions, since the Neumann case can be handled in a similar way. Formula 2.25) shows that the only remaining problem is to prove that the function $K_{1}(a)$ is differentiable with respect to $a$. To this end, we observe that

$$
K_{1}(a+h)=h \lim _{w_{1} \rightarrow 0^{+}} \frac{\partial \tilde{\psi}_{h}}{\partial w_{1}}\left(w_{1}, 0\right) .
$$

To check this, we note that $\phi_{D}(z, a)$ is analytical at $z=a+h$, and recalling (2.22), we can expand $\tilde{\phi}$ in terms of the variable $w^{2}=z-a-h$ to obtain

$$
\begin{aligned}
\tilde{\phi}(w ; a) & =\phi(z ; a)=\phi(a+h ; a)+C(z-a-h)+O\left((z-a-h)^{2}\right) \\
& =\tilde{\phi}(0 ; a)+C w^{2}+O\left(w^{4}\right),
\end{aligned}
$$

whence

and we eventually derive

$$
\frac{\partial \widetilde{\phi}_{D}}{\partial w_{1}}(0 ; a)=0
$$

$$
\begin{aligned}
\left.\frac{\partial}{\partial w_{1}}\right|_{w=0} h \tilde{\psi}_{h}(w) & =\left.\frac{\partial}{\partial w_{1}}\right|_{w=0}[\tilde{\phi}(w ; a+h)-\tilde{\phi}(w ; a)] \\
& =\left.\frac{\partial}{\partial w_{1}}\right|_{w=0} \tilde{\phi}(w ; a+h)=K_{1}(a+h),
\end{aligned}
$$


where (2.22) has been used again with $a$ replaced by $a+h$ there. Next we use the Poisson representation formula in the variable $w$ (cf. (2.24) ) as well as (2.22) to obtain

$\tilde{\psi}_{h}(w)=-\frac{1}{h \pi} \int_{-\sqrt{h}}^{\sqrt{h}} \frac{w_{1}\left[K_{1}(a) \sqrt{h-\eta^{2}}+K_{3}(a)\left(h-\eta^{2}\right)^{3 / 2}+O\left(\left(h-\eta^{2}\right)^{2}\right)\right]}{w_{1}^{2}+\left(w_{2}-\eta\right)^{2}} d \eta$.

Note that the term $K_{2}(a) \operatorname{Im}\left(h-\eta^{2}\right) \equiv 0$ gives no contribution to the integral. If we now take the derivative with respect to $w_{1}$ in (2.30) and then integrate by parts, we deduce that

$$
\begin{aligned}
& \frac{\partial \tilde{\psi}_{h}}{\partial w_{1}}\left(w_{1}, 0\right) \\
& =\frac{1}{h \pi} \int_{-\sqrt{h}}^{\sqrt{h}} \frac{\eta\left[K_{1}(a) \eta\left(h-\eta^{2}\right)^{-1 / 2}+3 K_{3}(a) \eta\left(h-\eta^{2}\right)^{1 / 2}+O\left(\eta\left(h-\eta^{2}\right)\right)\right]}{w_{1}^{2}+\eta^{2}} d \eta .
\end{aligned}
$$

Letting $w_{1} \rightarrow 0$ yields

$$
\lim _{w_{1} \rightarrow 0^{+}} \frac{\partial \tilde{\psi}_{h}}{\partial w_{1}}\left(w_{1}, 0\right) \underset{\eta=\bar{\eta} \sqrt{h}}{=} \frac{1}{h \pi} \int_{-1}^{1}\left[\frac{K_{1}(a)}{\sqrt{h} \sqrt{1-\bar{\eta}^{2}}}+3 \sqrt{h} K_{3}(a) \sqrt{1-\bar{\eta}^{2}}\right] \sqrt{h} d \bar{\eta} .
$$

Using (2.29) we can write

$$
K_{1}(a+h)=K_{1}(a)+\frac{3}{2} h K_{3}(a)
$$

and we eventually obtain

$$
\lim _{h \rightarrow 0} \frac{K_{1}(a+h)-K_{1}(a)}{h}=\frac{3}{2} K_{3}(a) .
$$

Proof of Theorem 2.1. Suppose that $u$ is given by (2.20). Standard computations yield

$$
\begin{aligned}
\square u & =\left(\frac{\partial^{2} \phi}{\partial a^{2}}(\dot{\lambda}(\tau))^{2}+\frac{\partial \phi}{\partial a} \ddot{\lambda}(\tau)\right)\left(\left(\frac{\partial \tau}{\partial t}\right)^{2}-|\nabla \tau|^{2}\right)+\frac{\partial \phi}{\partial a} \dot{\lambda}(\tau)\left(\frac{\partial^{2} \tau}{\partial t^{2}}-\Delta \tau\right) \\
(2.31)-2 \dot{\lambda}(\tau) & \nabla\left(\frac{\partial \phi}{\partial a}\right) \cdot \nabla \tau-\Delta \phi .
\end{aligned}
$$

Differentiating (2.19) with respect to $t$ and $x$ we obtain

$$
\begin{aligned}
& \frac{\partial \tau}{\partial t}=\frac{t-\tau}{A}, \\
& \nabla \tau=-\frac{\mathbf{x}-\lambda(\tau)}{A},
\end{aligned}
$$

where

$$
A=(t-\tau)-(x-\lambda(\tau)) \dot{\lambda}(\tau) .
$$

Then, using (2.32) and (2.33) as well as (2.19) we arrive at

$$
\left(\frac{\partial \tau}{\partial t}\right)^{2}-|\nabla \tau|^{2}=\frac{1}{A^{2}}\left((t-\tau)^{2}-|\mathbf{x}-\lambda(\tau)|^{2}\right)=0 .
$$


Differentiating (2.32) and (2.33) we see that

$$
\begin{aligned}
\frac{\partial^{2} \tau}{\partial t^{2}} & =\frac{1-\frac{\partial \tau}{\partial t}}{A}-\frac{(t-\tau)}{A^{2}} \frac{\partial A}{\partial t}, \\
\Delta \tau & =-\frac{\operatorname{div}(\mathbf{x}-\lambda(\tau))}{A}+\frac{1}{A^{2}}(\mathbf{x}-\lambda(\tau)) \cdot \nabla A .
\end{aligned}
$$

In order to compute $\frac{\partial A}{\partial t}$ and $\nabla A$ we just differentiate (2.34) and use (2.32) and (2.33), whence

$$
\begin{aligned}
& \frac{\partial A}{\partial t}=1-\frac{t-\tau}{A}+(\dot{\lambda})^{2} \frac{t-\tau}{A}-\ddot{\lambda} \frac{t-\tau}{A}(x-\lambda(\tau)) \\
& \nabla A=\frac{\mathbf{x}-\lambda(\tau)}{A}+\ddot{\lambda}(\tau) \frac{\mathbf{x}-\lambda(\tau)}{A}(x-\lambda(\tau))-\dot{\lambda}(\tau)-(\dot{\lambda}(\tau))^{2} \frac{\mathbf{x}-\lambda(\tau)}{A}
\end{aligned}
$$

On the other hand,

$$
\operatorname{div}(\mathbf{x}-\lambda(\tau))=2+\frac{\dot{\lambda}(\tau) \cdot(\mathbf{x}-\lambda(\tau))}{A} .
$$

Combining (2.36)-(2.40) we deduce after some computations that

$$
\square \tau=\frac{1}{A} .
$$

Plugging (2.3), (2.33), (2.35) and (2.41) in (2.31) we obtain

$$
\square u=\frac{\dot{\lambda}(\tau)}{A}\left\{\frac{\partial \phi}{\partial a}+2 \nabla\left(\frac{\partial \phi}{\partial a}\right) \cdot(\mathbf{x}-\lambda(\tau))\right\}+f(\mathbf{x}) .
$$

Using (2.21) we eventually derive

$$
\square u=f(\mathbf{x}),
$$

and the proof is complete.

We next point out the following consequence of Theorem 2.1.

Corollary 2.1. Suppose that $u$ is the solution of $\left(P_{D}\right)$ obtained in Theorem 2.1. Then

$$
u(\mathbf{x}, t) \sim \frac{K_{1}(\lambda(t))}{\sqrt{1-v^{2}}} \sqrt{r} \operatorname{Re}\left[\left(\cos \theta+i\left(1-v^{2}\right) \sin \theta+v \sqrt{1-(v \sin (\theta))^{2}}\right)^{1 / 2}\right],
$$

as $\mathbf{x} \rightarrow \lambda(t)$, where we write $z-\lambda(t)=r e^{i \theta}$ and $v=\dot{\lambda}(t)$.

Proof. It is just a consequence of (2.19)-(2.22). Note that (2.19) implies that for $\mathbf{x} \rightarrow \lambda(t), \tau(\mathbf{x}, t) \rightarrow t^{-}$. Expanding $\lambda(\tau(\mathbf{x}, t))$ by means of a Taylor series in (2.19) we see that

$$
|\mathbf{x}-\lambda(t)+\dot{\lambda}(t)(t-\tau(\mathbf{x}, t))|=t-\tau(\mathbf{x}, t)+o(t-\tau(\mathbf{x}, t)),
$$

as $\mathbf{x} \rightarrow \lambda(t)$. Using the polar representation $\mathbf{x}-\lambda(t)=|\mathbf{x}-\lambda(t)| e^{i \theta}$ we then obtain

$$
\left(\cos (\theta)+\dot{\lambda}(t) \frac{t-\tau(\mathbf{x}, t)}{|\mathbf{x}-\lambda(t)|}\right)^{2}+\sin ^{2}(\theta)=\frac{(t-\tau(\mathbf{x}, t))^{2}}{|\mathbf{x}-\lambda(t)|^{2}}(1+o(1)) \text { as } \mathbf{x} \rightarrow \lambda(t),
$$


whence, after some elementary computations we arrive at

$$
t-\tau(\mathbf{x}, t)=\frac{|\mathbf{x}-\lambda(t)|}{1-(\dot{\lambda}(t))^{2}}\left[\cos (\theta) \dot{\lambda}(t)+\sqrt{1-(\dot{\lambda}(t) \sin (\theta))^{2}}\right](1+o(1))
$$

as $\mathbf{x} \rightarrow \lambda(t)$. Using (2.20), (2.22) and the Taylor's expansion for $\lambda(t)$, and plugging in the resulting formula (2.44), we eventually derive (2.43).

In the case of Neumann boundary conditions we can show in a similar way

$u(\mathbf{x}, t) \sim C_{0}+\frac{K_{1}(\lambda(t))}{\sqrt{1-v^{2}}} \sqrt{r} \operatorname{Im}\left[\left(\cos \theta+i\left(1-v^{2}\right) \sin \theta+v \sqrt{1-(v \sin (\theta))^{2}}\right)^{1 / 2}\right]$,

when $\mathbf{x} \rightarrow \lambda(t)$. This is just another way of writing a classical formula for the asymptotic behavior of the solution for Mode III crack propagation that is derived in fracture mechanics. For example, after some elementary manipulations, formula (4.2.10) in Freund's book 4], can be recast in the form

$$
u(\mathbf{x}, t) \sim \frac{K(t)}{\sqrt{1-v^{2}}} \sqrt{r} \operatorname{Im}\left[\left(\cos \theta+i \sqrt{1-v^{2}} \sin \theta\right)^{1 / 2}\right] .
$$

It is also shown in 4 that, under suitable assumptions, $K(t)$ can be written as follows:

$$
K(t)=K_{1}(\lambda(t)) \sqrt{1-v} .
$$

Plugging (2.47) in (2.46), we obtain after some computations (2.45) when $C_{0}$ is set equal to zero there, a normalization condition.

An interesting fact about (2.27), (2.43) (respectively (2.28), (2.45) ) is that they provide a method for explicitly computing $\phi_{D}(x, a)$ (resp. $\phi_{N}(x, a)$ ) as soon as $\phi_{D}(x, 0)$ and $\phi_{N}(x, 0)$ are known. Indeed, for any given slit line, we can select a particular arbitrary motion $\lambda(t)$ proceeding at constant velocity, i.e., $\lambda(t)=$ $V t$ where $|V|<1$, for which $K_{1}(\lambda(t))$ in (2.43) (resp. (2.45) can be explicitly computed, a fact which will be recalled in the Appendix at the end of this paper. Since by formulae (2.43) (resp. (2.45) $K_{1}$ does not depend on the particular way in which motion is performed, $\phi_{D}(x, a)$ and $\phi_{N}(x, a)$ are thus determined in this manner.

\section{THE CASE OF ARBITRARY INITIAL DATA}

In this section we derive a representation formula that generalizes (2.20) for arbitrary initial values and time-dependent sources. As in the previous section, we use the notation $\Gamma_{a}:=\{(x, 0): x<a\}$ for a slit line with fixed endpoint. Let us consider the problem

$$
\begin{aligned}
\square u & =f(\mathbf{x}, t), \\
u(\mathbf{x}, 0) & =u_{0}(\mathbf{x}), \\
u_{t}(\mathbf{x}, 0) & =v_{0}(\mathbf{x}) .
\end{aligned}
$$

We assume $f \in C\left(\mathbb{R}^{2} \times \mathbb{R}^{+}\right), u_{0} \in C^{2}\left(\mathbb{R}^{2} \backslash \Gamma_{0}\right)$ and $v_{0} \in C^{1}\left(\mathbb{R}^{2} \backslash \Gamma_{0}\right)$, all supported in a ball of finite radius. This is just to avoid nonessential technicalities, but as will be apparent from our approach, it is possible to extend the forthcoming results to wider classes of data and sources. 
We consider both types of boundary conditions,

$$
u(\mathbf{x}, t)=0 \quad \mathbf{x} \in \Gamma(t)
$$

or

$$
\frac{\partial u}{\partial n}(\mathbf{x}, t)=0 \quad \mathbf{x} \in \Gamma(t)
$$

We shall continue to denote by $\left(P_{D}\right)$ (respectively by $\left.\left(P_{N}\right)\right)$ the problem consisting of (3.1)-(3.4) (respectively consisting of (3.1)-(3.3), (3.5)).

In the case of general data it is not true in general that the solutions of the problems $\left(P_{D}\right),\left(P_{N}\right)$ admit so simple a representation as that in (2.20). Nevertheless, it turns out that the structure of the solutions of such problems bears some similitude with that of the cases considered in the previous section. The main result of this section is the following.

Theorem 3.1. Suppose that $f, u_{0}, v_{0}$, are as above, and $\lambda \in C^{2}([0, \infty])$. Then, there exists a unique solution $u$ of problems $\left(P_{D}\right),\left(P_{N}\right)$ which belongs to

$$
C^{2}\left(\left(\mathbb{R}^{2} \times \mathbb{R}^{+}\right) \backslash \bigcup_{t>0}(\Gamma(t) \times\{t\})\right)
$$

and satisfies the following energy inequality:

$$
\int_{0}^{T} d t \int_{\mathbb{R}^{2} \backslash \Gamma(t)}\left(|\nabla u|^{2}+u_{t}^{2}\right) d x_{1} d x_{2}<\infty \quad \text { for any } T>0 .
$$

Moreover, the solution admits a representation formula given respectively by

$$
\begin{array}{ll}
u(\mathbf{x}, t)=\phi_{D}(\mathbf{x}, t ; \lambda(\tau(\mathbf{x}, t))) & \text { when } \\
u(\mathbf{x}, t)=\phi_{N}(\mathbf{x}, t ; \lambda(\tau(\mathbf{x}, t))) & \text { when holds, }
\end{array}
$$

where $\phi_{D}(\mathbf{x}, t ; a), \phi_{N}(\mathbf{x}, t ; a)$ are respectively given by (3.15) and (3.16) below.

We point out that existence results for quasi-static crack motion have been recently obtained in [3].

It is worth remarking that (3.6) cannot be satisfied by solutions that grow rapidly enough as $|\mathbf{x}| \rightarrow \infty$. For instance, the particular solutions $\phi_{N}(\mathbf{x} ; a)$ obtained in Lemma 2.1 are such that (2.12) holds. While a local integrability condition near the crack tip is satisfied (cf. (2.7)), it can be shown that (2.12) is actually incompatible with the global estimate (3.6). Nevertheless, due to the linearity of the problem, our argument below is readily extended so as to yield uniqueness for, say, any solution $u(\mathbf{x}, t)$ such that $u(\mathbf{x}, t)-\phi_{N}(\mathbf{x} ; 0)\left(\right.$ resp. $\left.u(\mathbf{x}, t)-\phi_{D}(\mathbf{x} ; 0)\right)$ satisfies (3.6).

Proof of Theorem 3.1. Existence and representation formula. We shall proceed by looking for solutions in the form (3.7), (3.8) to derive the conditions that such functions $\phi_{D}, \phi_{N}$ should satisfy. The proof of the existence part would then follow upon constructing suitable functions $\phi_{D}, \phi_{N}$, verifying such relations.

For simplicity let us denote by $\phi$ either $\phi_{D}$ or $\phi_{N}$, that we assume for the moment to exist and to be as smooth as required in order for the following computations to make sense. Let us denote by $u$ the function given either by (3.7) or (3.8). 
Elementary computations then yield

$$
\begin{aligned}
\square u & =\square \phi+2 \dot{\lambda}(\tau)\left\{\frac{\partial \tau}{\partial t} \frac{\partial^{2} \phi}{\partial t \partial a}-\nabla_{\mathbf{x}}\left(\frac{\partial \phi}{\partial a}\right) \cdot \nabla \tau+\frac{1}{2} \frac{\partial \phi}{\partial a} \square \tau\right\} \\
& +\left(\frac{\partial^{2} \phi}{\partial a^{2}}(\dot{\lambda}(\tau))^{2}+\frac{\partial \phi}{\partial a} \ddot{\lambda}(\tau)\right)\left(\left(\frac{\partial \tau}{\partial t}\right)^{2}-|\nabla \tau|^{2}\right) .
\end{aligned}
$$

Note that (2.35) implies that the last term in (3.9) vanishes. We now require that

$$
\square \phi=f(\mathbf{x}, t)
$$

and

$$
(t-\tau) \frac{\partial^{2} \phi}{\partial t \partial a}+\nabla_{\mathbf{x}}\left(\frac{\partial \phi}{\partial a}\right) \cdot(\mathbf{x}-a(1,0))+\frac{1}{2} \frac{\partial \phi}{\partial a}=0
$$

Summarizing, we need any of the functions $\phi_{D}, \phi_{N}$ in Theorem 3.1 to solve (3.10) and (3.11) as well as the boundary conditions (3.4) or (3.5), respectively. The rest of the proof consists in constructing $\phi_{D}, \phi_{N}$ satisfying these conditions.

We now look for functions $\phi$ in the form

$$
\frac{\partial \phi}{\partial a}=h(t-r, a) r^{-1 / 2} G(\theta),
$$

where $r=r(\mathbf{x}, a)=|\mathbf{x}-\mathbf{a}|, h$ is a function to be determined, and we denote by $\theta=\theta(\mathbf{x}, a)$ the angle between the vectors $\mathbf{x}-\mathbf{a}$ and $(1,0)$.

Differentiating on (3.10) we obtain that $\frac{\partial \phi}{\partial a}$ solves the wave equation in $\mathbb{R}^{2} \backslash \Gamma_{a}$ with Dirichlet or Neumann boundary conditions for $\frac{\partial \phi_{D}}{\partial a}, \frac{\partial \phi_{N}}{\partial a}$, respectively. Then, plugging (3.12) into the wave equation, it follows after some computations that

$$
G^{\prime \prime}(\theta)+\frac{1}{4} G(\theta)=0
$$

with boundary conditions $G(\theta)=0$ when $\theta= \pm \pi$ in the Dirichlet case. Hence

$$
\frac{\partial \phi_{D}}{\partial a}=h(t-r, a) r^{-1 / 2} \cos \left(\frac{\theta}{2}\right)
$$

and $G^{\prime}(\theta)=0$ when $\theta= \pm \pi$ for Neumann boundary conditions. One then has that

$$
\frac{\partial \phi_{N}}{\partial a}=h(t-r, a) r^{-1 / 2} \sin \left(\frac{\theta}{2}\right) .
$$

On integrating (3.13), (3.14) we obtain

$$
\phi_{D}(\mathbf{x}, t ; a)=\phi_{D}(\mathbf{x}, t ; 0)+\int_{0}^{a} h(t-r(\mathbf{x}, \xi), \xi)(r(\mathbf{x}, \xi))^{-1 / 2} \cos \left(\frac{\theta(\mathbf{x}, \xi)}{2}\right) d \xi,
$$

$$
\phi_{N}(\mathbf{x}, t ; a)=\phi_{N}(\mathbf{x}, t ; 0)+\int_{0}^{a} h(t-r(\mathbf{x}, \xi), \xi)(r(\mathbf{x}, \xi))^{-1 / 2} \sin \left(\frac{\theta(\mathbf{x}, \xi)}{2}\right) d \xi .
$$

We now proceed to identify the functions appearing on the right-hand side of the formulae above. Concerning $\phi_{D}(\mathbf{x}, t ; 0)$ we require that it should solve the following 
problem:

$$
\begin{aligned}
\square z & =f \quad \text { in } \quad \mathbb{R}^{2} \backslash \Gamma(0), t>0, \\
z(\mathbf{x}, t) & =0 \quad \text { in } \Gamma(0), \\
z(\mathbf{x}, 0) & =u_{0}(\mathbf{x}), \quad \frac{\partial z}{\partial t}(\mathbf{x}, 0)=v_{0}(\mathbf{x}),
\end{aligned}
$$

whereas in turn $\phi_{N}(\mathbf{x}, t ; 0)$ is taken as a solution to

$$
\begin{aligned}
\square z & =f \quad \text { in } \quad \mathbb{R}^{2} \backslash \Gamma(0), t>0, \\
\frac{\partial z}{\partial x_{2}}(\mathbf{x}, t) & =0 \quad \text { in } \Gamma(0), \\
z(\mathbf{x}, 0) & =u_{0}(\mathbf{x}), \quad \frac{\partial z}{\partial t}(\mathbf{x}, 0)=v_{0}(\mathbf{x}) .
\end{aligned}
$$

We note that (3.17) and (3.18) can be solved by classical methods. For convenience of the reader we sketch the corresponding argument in an Appendix at the end of the paper. Concerning function $h$, consider for definiteness the Dirichlet case. On imposing (3.4) on (3.15) we readily obtain

$$
\int_{0}^{x_{1}} h\left(t-\left(x_{1}-\xi\right), \xi\right)\left(x_{1}-\xi\right)^{-1 / 2} d \xi=-\phi_{D}\left(\left(x_{1}, 0\right), t ; 0\right), \quad \text { for } 0 \leq x_{1}<a .
$$

This equation can be solved by standard Laplace transform techniques to give

$$
h(t, a)=-\frac{1}{\pi} \int_{0}^{a}\left(\frac{\partial \phi_{D}}{\partial t}+\frac{\partial \phi_{D}}{\partial x_{1}}\right)\left(\left(x_{1}-\zeta, 0\right), t-\zeta ; 0\right) \frac{d \zeta}{\sqrt{\zeta}},
$$

and a similar result can be obtained for the case of Neumann conditions.

To conclude with this part of the proof, we merely observe that the second term on the right in formula (3.15), denoted now by $\sigma(\mathbf{x}, t)$, is such that

$$
\square \sigma=0 .
$$

The case of Neumann conditions is similarly dealt with.

Proof of Theorem 3.1. Uniqueness. Let us introduce the change of variables:

$$
\left(x_{1}, x_{2}, t\right) \rightarrow\left(\bar{x}_{1}, \bar{x}_{2}, t\right) \equiv\left(x_{1}-\lambda(t), x_{2}, t\right) .
$$

In this way, if $u$ solves (1.1), we have that the function $\bar{u}$ given by

$$
\bar{u}\left(x_{1}-\lambda(t), x_{2}, t\right)=u\left(x_{1}, x_{2}, t\right)
$$

satisfies the following equation:

$$
\frac{\partial^{2} \bar{u}}{\partial t^{2}}=\left(1-v^{2}\right) \frac{\partial^{2} \bar{u}}{\partial \bar{x}_{1}^{2}}+2 v \frac{\partial^{2} \bar{u}}{\partial t \partial \bar{x}_{1}}+\frac{\partial^{2} \bar{u}}{\partial \bar{x}_{2}^{2}}+\dot{v} \frac{\partial \bar{u}}{\partial \bar{x}_{1}},
$$

where $v=\dot{\lambda}(t)$. If we now define

$$
w_{1}=\frac{\partial \bar{u}}{\partial t}, \quad w_{2}=\sqrt{1-v^{2}} \frac{\partial \bar{u}}{\partial \bar{x}_{1}}, \quad w_{3}=\frac{\partial \bar{u}}{\partial \bar{x}_{2}},
$$

we can write (3.20) as follows:

$$
\frac{\partial \vec{w}}{\partial t}+A(t) \frac{\partial \vec{w}}{\partial \bar{x}_{1}}+B(t) \frac{\partial \vec{w}}{\partial \bar{x}_{2}}+C(t) \vec{w}=0,
$$


where $\vec{w}=\left(w_{1}, w_{2}, w_{3}\right)$ and

$$
\begin{aligned}
A & =\left(\begin{array}{ccc}
-2 v & -\sqrt{1-v^{2}} & 0 \\
-\sqrt{1-v^{2}} & 0 & 0 \\
0 & 0 & 0
\end{array}\right), \\
B & =\left(\begin{array}{ccc}
0 & 0 & -1 \\
0 & 0 & 0 \\
-1 & 0 & 0
\end{array}\right), \quad C=\left(\begin{array}{ccc}
0 & -\frac{\dot{v}}{\sqrt{1-v^{2}}} & 0 \\
0 & \frac{v v}{1-v^{2}} & 0 \\
0 & 0 & 0
\end{array}\right) .
\end{aligned}
$$

Note that $A, B$ are symmetric and then (3.22) is a symmetric hyperbolic system (see for instance F. John [9]). We now argue by contradiction, and assume that there exist two different solutions, $u_{1}$ and $u_{2}$, of the problem under consideration. Let $\vec{w}_{1}, \vec{w}_{2}$ be the functions obtained from $u_{1}, u_{2}$ via (3.21), and set $\vec{w}=\vec{w}_{1}-\vec{w}_{2}$. As usual when dealing with hyperbolic systems, we shall show that $\vec{w} \equiv 0$ in suitable cone-like regions corresponding to the domain of influence of the data.

Consider a bounded region $\Omega$ in the space-time variables. If we make the scalar product of identity (3.22) by $\vec{w}$ and then integrate by parts in $\Omega$, we find that

$$
\iint_{\partial \Omega} \xi\langle A(t) \vec{w}, \vec{w}\rangle+\eta\langle B(t) \vec{w}, \vec{w}\rangle+\tau\langle\vec{w}, \vec{w}\rangle=\iiint_{\Omega}\langle D(t) \vec{w}, \vec{w}\rangle,
$$

where $(\xi, \eta, \tau)$ is the unit vector normal to $\partial \Omega$ and $D=-\left(C+C^{T}\right)$ is given by

$$
D(t)=\left(\begin{array}{ccc}
0 & \frac{\dot{v}}{\sqrt{1-v^{2}}} & 0 \\
\frac{\dot{v}}{\sqrt{1-v^{2}}} & -\frac{2 v \dot{v}}{1-v^{2}} & 0 \\
0 & 0 & 0
\end{array}\right) .
$$

Equation (3.24) is the so-called energy identity of the symmetric hyperbolic system.

Now consider space-time regions defined in the following way:

$$
\Omega=\left\{\left(\bar{x}_{1}, \bar{x}_{2}, t\right):\left(\bar{x}_{1}, \bar{x}_{2}\right) \in R(t) \subset \mathbb{R}^{2} \backslash \Gamma(0), 0 \leq t \leq T\right\},
$$

for some value $T>0$. The boundary of $\Omega$ in $\mathbb{R}^{2} \times \mathbb{R}$ can be split into the following sets:

$$
\begin{aligned}
S_{1} & =\left\{\left(\bar{x}_{1}, \bar{x}_{2}, 0\right):\left(\bar{x}_{1}, \bar{x}_{2}\right) \in R(0)\right\} \text { (floor), } \\
S_{2} & =\left\{\left(\bar{x}_{1}, \bar{x}_{2}, T\right):\left(\bar{x}_{1}, \bar{x}_{2}\right) \in R(T)\right\} \text { (roof), } \\
S_{3} & =\partial \Omega \cap\{\Gamma(0) \times[0, T]\} \quad \text { (the vertical wall), } \\
S & =\partial \Omega \backslash\left(S_{1} \cup S_{2} \cup S_{3}\right) .
\end{aligned}
$$

On $S_{3}$ we have that $(\xi, \eta, \tau)^{ \pm}=(0, \mp 1,0)$ (taking into account both sides of this wall), and then

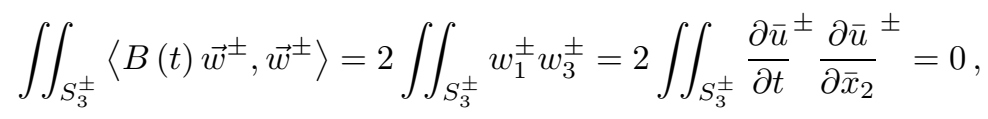

the last identity being a consequence of (3.23), (3.21) and the Dirichlet or Neumann boundary conditions imposed over $\Gamma$. Assume for the moment that the next inequality holds:

$$
\xi\langle A(t) \vec{w}, \vec{w}\rangle+\eta\langle B(t) \vec{w}, \vec{w}\rangle+\tau\langle\vec{w}, \vec{w}\rangle \geq 0 \quad \text { on } S .
$$


Taking into account that $(\xi, \eta, \tau)=(0,0,-1)$ for $S_{1}$ and $(\xi, \eta, \tau)=(0,0,1)$ for $S_{2}$ together with (3.25), it then follows from the energy integral (3.24) that

$$
\iint_{S_{2}}\langle\vec{w}, \vec{w}\rangle_{t=T} \leq \iint_{S_{1}}\langle\vec{w}, \vec{w}\rangle_{t=0}+\int_{0}^{T}\left(\iint_{R(t)}|\langle D \vec{w}, \vec{w}\rangle|\right) d t .
$$

On the other hand we have that, for fixed $T>0$, then $v<1-\varepsilon$ for some $\varepsilon>0$ and all $t \leq T$. There exists $M(T)>0$ such that

$$
|\langle D(t) \vec{w}, \vec{w}\rangle| \leq M(T)\langle\vec{w}, \vec{w}\rangle, \quad \text { for } t \leq T .
$$

If we write

$$
E(t)=\iint_{R(t)}\langle\vec{w}, \vec{w}\rangle d \bar{x}_{1} d \bar{x}_{2}
$$

we have that (cf. (3.26) $)$

$$
E(t) \leq E(0)+M(T) \int_{0}^{t} E(\zeta) d \zeta
$$

whence

$$
E(t) \leq E(0) e^{M(T) t} .
$$

Uniqueness is then derived from the fact that $E(0)=0$ by assumption.

To conclude this part of the proof, we have yet to check that we can select the region $\Omega$ in such a way that (3.25) holds in $S$. If we define the $3 \times 3$ matrix

$$
H(t, \xi, \eta, \tau):=\xi A(t)+\eta B(t)+\tau I,
$$

where $I$ is the identity, then (3.25) can be written as follows:

$$
\langle H(t, \xi, \eta, \tau) \vec{w}, \vec{w}\rangle \geq 0 .
$$

Note that

$$
H(t, \xi, \eta, \tau)=\left(\begin{array}{ccc}
\tau-2 v \xi & -\xi \sqrt{1-v^{2}} & -\eta \\
-\xi \sqrt{1-v^{2}} & \tau & 0 \\
-\eta & 0 & \tau
\end{array}\right) .
$$

Let us take $\Omega$ bounded by a cone whose normal has a positive component in the $t$ direction $(\tau>0)$. Such a cone can be described by the equation

$$
b\left(\bar{x}_{1}^{2}+\bar{x}_{2}^{2}\right)-(L-t)^{2}=0
$$

for some positive constants $L$ and $b$. In this way, for each fixed $t<L$, we have that

$$
R(t)=\left\{\left(\bar{x}_{1}, \bar{x}_{2}\right): \bar{x}_{1}^{2}+\bar{x}_{2}^{2} \leq \frac{(L-t)^{2}}{b}\right\},
$$

and the normal vector for the lower part of the cone surface is given by

$$
(\xi, \eta, \tau)=\frac{1}{\sqrt{b+1}}(\sqrt{b} \cos \theta, \sqrt{b} \sin \theta, 1),
$$

where we parameterized by $\theta$ by means of

$$
\left(\bar{x}_{1}, \bar{x}_{2}\right)=\frac{L-t}{\sqrt{b}}(\cos \theta, \sin \theta),
$$

for $\left(\bar{x}_{1}, \bar{x}_{2}\right)$ in $S$ and $t<L$. We can easily see that if we take the constant $b$ close enough to 0 (i.e. the cone is wider), then $\xi, \eta$ are near zero, and $\tau$ is close to the value 1 . Then $H$ is close to the identity matrix (cf. (3.28) ) and (3.25) is thus 
achieved. Note that we can take $L>T$ as large as we want in order to include any bounded region in space-time variables within such a cone.

Let us elaborate a bit on formulae (3.15), (3.16). To begin with, we will rewrite them using complex variable notation. We then have that

$$
\begin{aligned}
& \phi_{D}(\mathbf{x}, t ; a)=\phi_{D}(\mathbf{x}, t ; 0)+\int_{0}^{a} h(t-|\mathbf{x}-\xi|, \xi) \operatorname{Re}\left(\frac{1}{\sqrt{\mathbf{x}-\xi}}\right) d \xi, \\
& \phi_{N}(\mathbf{x}, t ; a)=\phi_{N}(\mathbf{x}, t ; 0)-\int_{0}^{a} h(t-|\mathbf{x}-\xi|, \xi) \operatorname{Im}\left(\frac{1}{\sqrt{\mathbf{x}-\xi}}\right) d \xi,
\end{aligned}
$$

where we set $\mathbf{x}=x_{1}+i x_{2}, \xi=\xi_{1}+i \xi_{2}$ in quantities containing complex arguments. Integrating by parts in (3.29) we obtain

$\phi_{D}(\mathbf{x}, t ; a)=\phi_{D}(\mathbf{x}, t ; 0)-2 h(t-|\mathbf{x}-\mathbf{a}|, a) \operatorname{Re}(\sqrt{\mathbf{x}-\mathbf{a}})+2 h(t-|\mathbf{x}|, a) \operatorname{Re}(\sqrt{\mathbf{x}})$

$$
+2 \int_{0}^{a} \frac{d}{d \xi}[h(t-|\mathbf{x}-\xi|, \xi)] \frac{(\xi-\mathbf{x}) \cdot \mathbf{e}}{|\xi-\mathbf{x}|} \operatorname{Re}(\sqrt{\mathbf{x}-\xi}) d \xi
$$

where $\mathbf{e}=(1,0)$. An analogous formula holds for the case of Neumann boundary conditions. Using (3.31), as well as the fact that $\phi_{D}(\mathbf{a}, t ; a)=0$ (by the Dirichlet conditions), and since we are assuming that $h$ is smooth enough, we obtain the asymptotics

$$
\phi_{D}(\mathbf{x}, t ; a) \sim-2 h(t, a) \operatorname{Re}(\sqrt{\mathbf{x}-\mathbf{a}}) \text { as } \mathbf{x} \rightarrow \mathbf{a},
$$

and in an analogous manner

$$
\phi_{N}(\mathbf{x}, t ; a) \sim C_{0}(a)+2 h(t, a) \operatorname{Im}(\sqrt{\mathbf{x}-\mathbf{a}}) \text { as } \mathbf{x} \rightarrow \mathbf{a},
$$

where

$$
C_{0}(a)=\phi_{N}(\mathbf{a}, t ; 0)-\int_{0}^{a} h(t-|\mathbf{a}-\xi|, \xi) \operatorname{Im}\left(\frac{1}{\sqrt{\mathbf{a}-\xi}}\right) d \xi .
$$

Note that (3.32), (3.33) mean that the function $h(t, \lambda(t)$ ) is essentially (up to multiplicative constants) the coefficient that describes the asymptotics of $u$, the solution of (1.1)-(1.4) near $\lambda(t)$ (cf. (1.5)). More precisely, arguing exactly as in Corollary 2.1, it turns out that $u$, the solution of $\left(P_{D}\right)$, satisfies

$$
u(\mathbf{x}, t) \sim-\frac{2 h(t, \lambda(t))}{\sqrt{1-v^{2}}} \sqrt{r} \operatorname{Re}\left[\left(\cos \theta+i\left(1-v^{2}\right) \sin \theta+v \sqrt{1-(v \sin (\theta))^{2}}\right)^{1 / 2}\right]
$$

when $r=|\mathbf{x}-\lambda(t)| \rightarrow 0$.

On the other hand $u$, the solution of $\left(P_{N}\right)$, behaves as

$u(\mathbf{x}, t) \sim C_{0}+\frac{2 h(t, \lambda(t))}{\sqrt{1-v^{2}}} \sqrt{r} \operatorname{Im}\left[\left(\cos \theta+i\left(1-v^{2}\right) \sin \theta+v \sqrt{1-(v \sin (\theta))^{2}}\right)^{1 / 2}\right]$ when $r \rightarrow 0$.

Actually (3.34) provides another method of finding $h(t, a)$ as an alternative to (3.19). Since the local asymptotics of $u$ near $\lambda(t)$ depend only on $\lambda(t), \dot{\lambda}(t)$, if we derive an explicit representation formula for a simple choice of the function $\lambda(t)$, we could then use (3.7), (3.8) to obtain similar formulae for arbitrary choices of $\lambda(t)$. In particular, using (3.34), (3.35) we could compute $h(t)$. A choice of $\lambda(t)$ which is simple to analyse $u$ is $\lambda(t)=V t$ with $0<V<1$, since in that case the resulting boundary value problem has constant coefficients, and the corresponding 


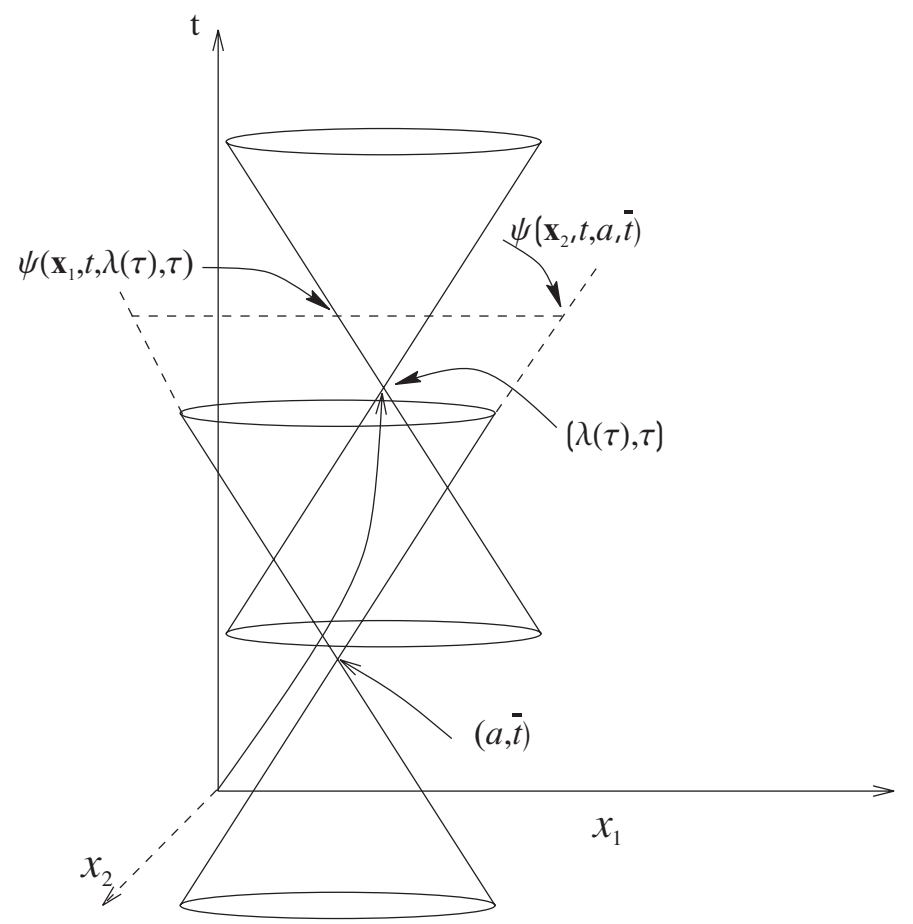

Figure 2. The geometric structure of solutions. Here $\psi$ stands for $\phi_{D}$ or $\phi_{N}$ in each case.

solutions can be derived by means of the classical Wiener-Hopf method (see the Appendix). It then follows that (cf. (A.22) $)$

$$
\begin{aligned}
h(t, a)= & -\frac{\sqrt{1-\frac{a}{t}}}{\left(1-\left(\frac{a}{t}\right)^{2}\right)^{1 / 4}} \frac{e^{-\frac{i \pi}{4}}}{\sqrt{2} \pi^{2}} \\
& \times \int_{\tau-i \infty}^{\tau+i \infty} e^{\frac{t z}{\sqrt{1-\left(\frac{a}{t}\right)^{2}}}}\left[\int_{\mathbb{R}} \sqrt{\xi-i z} \psi(\xi, z) d \xi\right] d z, \quad \tau>0,
\end{aligned}
$$

where $\psi$ depends on the initial data and is given in the Appendix (see formula (A.19) ). A similar formula holds for $\left(P_{N}\right)$ and is given by (cf. (A.23)

$$
\begin{aligned}
h(t, a)= & \frac{\sqrt{1+\frac{a}{t}}}{\left(1-\left(\frac{a}{t}\right)^{2}\right)^{1 / 4}} \frac{e^{-\frac{i \pi}{4}}}{\sqrt{2} \pi^{2} i} \\
& \times \int_{\tau-i \infty}^{\tau+i \infty} e^{\frac{t z}{\sqrt{1-\left(\frac{a}{t}\right)^{2}}}}\left[\int_{\mathbb{R}} \sqrt{\xi+i z} \psi(\xi, z) d \xi\right] d z \quad \tau>0 .
\end{aligned}
$$

We conclude this section by remarking that the representation formulae (3.7), (3.8) provide geometric insight into the form of the solutions of problems $\left(P_{D}\right)$, $\left(P_{N}\right)$. Indeed (see Figure 2), consider the family of cones $|\mathbf{x}-\mathbf{a}|=t-\bar{t}$ for $\mathbf{a}=(a, 0)$, and $a, \bar{t}$ arbitrary. For any given slit motion $\lambda(t)$, points as $\left(\mathbf{x}_{2}, t\right)$ cannot be reached by cones with vertex at the graph of $\lambda(t)$. Therefore, $u\left(\mathbf{x}_{2}, t\right)=$ $\psi\left(\mathbf{x}_{2}, t ; 0\right)$, where $\psi(\mathbf{x}, t ; 0)$ stands for the solutions of problems (3.17) or (3.18). 
On the other hand, a point like $\left(\mathbf{x}_{1}, t\right)$ lies over the cone centered at $(\lambda(\tau), \tau)$, so that $u\left(\mathbf{x}_{1}, t\right)=\psi\left(\mathbf{x}_{1}, t ; \lambda\left(\tau\left(\mathbf{x}_{1}, t\right)\right)\right)$. Note that, when drawing the figure, we have specified in each case, as an argument in function $\psi$, the vertex of the cone we are referring to.

\section{Appendix: Computation of $h(t)$ FOR A CRACK AT CONSTANT SPEED}

For convenience of the reader, in this Appendix we solve explicitly $\left(P_{D}\right),\left(P_{N}\right)$ in the case of cracks propagating at constant subsonic speed $V$. More precisely we consider the following problems:

$$
\begin{aligned}
\square u(\mathbf{x}, t) & =f(\mathbf{x}, t) \text { in } \mathbb{R}^{2} \backslash \Gamma_{V t}, \\
u\left(x_{1}, 0, t\right) & =0 \text { in } x_{1}<V t, \\
u(\mathbf{x}, 0) & =u_{0}(x) \text { in } \mathbb{R}^{2} \backslash \Gamma_{0}, \\
u_{t}(\mathbf{x}, 0) & =v_{0}(x) \text { in } \mathbb{R}^{2} \backslash \Gamma_{0} .
\end{aligned}
$$

Also,

$$
\begin{aligned}
\square u(\mathbf{x}, t) & =f(\mathbf{x}, t) \text { in } \mathbb{R}^{2} \backslash \Gamma_{V t}, \\
\frac{\partial u}{\partial x_{2}}\left(x_{1}, 0, t\right) & =0 \text { in } x_{1}<V t, \\
u(\mathbf{x}, 0) & =u_{0}(x) \text { in } \mathbb{R}^{2} \backslash \Gamma_{0}, \\
u_{t}(\mathbf{x}, 0) & =v_{0}(x) \text { in } \mathbb{R}^{2} \backslash \Gamma_{0} .
\end{aligned}
$$

We want to compute explicitly the coefficients in (2.43), (3.34) in terms of $f, u_{0}, v_{0}$. Solving (A.1), A.2 is achieved by application of the classical WienerHopf method (cf. for instance [13]), and we will sketch below the main steps in the argument for completeness. We will focus on problem (A.1) since the details for (A.2) are similar.

Note that since the tip of the crack propagates at constant velocity, we can reduce (A.1), (A.2) to problems in fixed domains by means of the Lorentz transformation

$$
\begin{aligned}
x_{1}^{\prime} & =\frac{x_{1}-V t}{\sqrt{1-V^{2}}}, \\
x_{2}^{\prime} & =x_{2}, \\
t^{\prime} & =\frac{t-V x_{1}}{\sqrt{1-V^{2}}},
\end{aligned}
$$

that changes (A.1) into

$$
\begin{aligned}
\square_{\left(\mathbf{x}^{\prime}, t^{\prime}\right)} w\left(\mathbf{x}^{\prime}, t^{\prime}\right) & =g\left(\mathbf{x}^{\prime}, t^{\prime}\right) \text { in } \mathbb{R}^{2} \backslash \Gamma_{0}, \\
w\left(x_{1}^{\prime}, 0, t^{\prime}\right) & =0 \text { in } x_{1}^{\prime}<0, \\
w\left(\mathbf{x}^{\prime}, 0\right) & =w_{0}\left(\mathbf{x}^{\prime}\right) \text { in } \mathbb{R}^{2} \backslash \Gamma_{0}, \\
w_{t}\left(\mathbf{x}^{\prime}, 0\right) & =z_{0}\left(\mathbf{x}^{\prime}\right) \text { in } \mathbb{R}^{2} \backslash \Gamma_{0},
\end{aligned}
$$


where

$$
\begin{aligned}
w\left(\mathbf{x}^{\prime}, t^{\prime}\right) & =u(\mathbf{x}, t)=u\left(\frac{x_{1}^{\prime}+V t^{\prime}}{\sqrt{1-V^{2}}}, x_{2}^{\prime}, \frac{t^{\prime}+V x_{1}^{\prime}}{\sqrt{1-V^{2}}}\right), \\
g\left(\mathbf{x}^{\prime}, t^{\prime}\right) & =f(\mathbf{x}, t)=f\left(\frac{x_{1}^{\prime}+V t^{\prime}}{\sqrt{1-V^{2}}}, x_{2}^{\prime}, \frac{t^{\prime}+V x_{1}^{\prime}}{\sqrt{1-V^{2}}}\right), \\
w_{0}\left(\mathbf{x}^{\prime}\right) & =u_{0}(\mathbf{x})=u_{0}\left(\frac{x_{1}^{\prime}}{\sqrt{1-V^{2}}}, x_{2}^{\prime}\right), \\
z_{0}\left(\mathbf{x}^{\prime}\right) & =\frac{1}{\sqrt{1-V^{2}}}\left[v_{0}\left(\frac{x_{1}^{\prime}}{\sqrt{1-V^{2}}}, x_{2}^{\prime}\right)+V \frac{\partial u_{0}}{\partial x_{1}}\left(\frac{x_{1}^{\prime}}{\sqrt{1-V^{2}}}, x_{2}^{\prime}\right)\right] .
\end{aligned}
$$

The advantage of (A.6)-A.9 compared with (A.1) is that in A.6 - A.9 we are already able to use the classical Wiener-Hopf approach. To this end, as a first step, we decompose $w$ in symmetric and skew-symmetric parts with respect to the $x_{1}$ axis. Given $\varphi(\mathbf{x}, t)$ arbitrary we write

$$
\begin{aligned}
& \varphi_{E}(\mathbf{x}, t)=\frac{1}{2}\left(\varphi\left(x_{1}, x_{2}, t\right)+\varphi\left(x_{1},-x_{2}, t\right)\right), \\
& \varphi_{O}(\mathbf{x}, t)=\frac{1}{2}\left(\varphi\left(x_{1}, x_{2}, t\right)-\varphi\left(x_{1},-x_{2}, t\right)\right) .
\end{aligned}
$$

Note that

$$
\begin{aligned}
& \square_{\left(\mathbf{x}^{\prime}, t^{\prime}\right)} w_{E}\left(\mathbf{x}^{\prime}, t^{\prime}\right)=g_{E}\left(\mathbf{x}^{\prime}, t^{\prime}\right) \text { in } \mathbb{R}^{2} \backslash \Gamma_{0}, \\
& \square_{\left(\mathbf{x}^{\prime}, t^{\prime}\right)} w_{O}\left(\mathbf{x}^{\prime}, t^{\prime}\right)=g_{O}\left(\mathbf{x}^{\prime}, t^{\prime}\right) \text { in } \mathbb{R}^{2} \backslash \Gamma_{0} .
\end{aligned}
$$

On the other hand $w_{O}$ satisfies the boundary condition $w_{O}=0$ in $x_{2}^{\prime}=0$ and then $w_{O}$ behaves linearly as $|\mathbf{x}| \rightarrow 0$. Therefore, since we are interested in computing the asymptotics of $w$ until order $O\left(|\mathbf{x}|^{1 / 2}\right)$, we can ignore $w_{O}$ and restrict our analysis to $w_{E}$. This last function satisfies A.10 as well as the boundary condition (A.7), and due to its symmetry it is such that

$$
\frac{\partial w_{E}}{\partial x_{2}^{\prime}}\left(x_{1}^{\prime}, 0, t^{\prime}\right)=0, x_{1}^{\prime}>0 .
$$

To proceed further, we introduce the Laplace transform of $w_{E}, g_{E}$ on $t^{\prime}$ and the Fourier transform on $x_{1}^{\prime}$ :

$$
W\left(k, x_{2}^{\prime}, z\right) \equiv \frac{1}{\sqrt{2 \pi}} \int_{0}^{\infty}\left[\int_{-\infty}^{\infty} w_{E}\left(x_{1}^{\prime}, x_{2}^{\prime}, t^{\prime}\right) e^{-z t^{\prime}} e^{-i k x_{1}^{\prime}} d x_{1}^{\prime}\right] d t^{\prime}, \quad \operatorname{Re} z>0
$$

$$
G\left(k, x_{2}^{\prime}, z\right) \equiv \frac{1}{\sqrt{2 \pi}} \int_{0}^{\infty}\left[\int_{-\infty}^{\infty} g_{E}\left(x_{1}^{\prime}, x_{2}^{\prime}, t^{\prime}\right) e^{-z t^{\prime}} e^{-i k x_{1}^{\prime}} d x_{1}^{\prime}\right] d t^{\prime}, \quad \operatorname{Re} z>0,
$$

as well as the standard Fourier transforms of $u_{0, E}, v_{0, E}$, that will be denoted by $\hat{u}_{0, E}\left(k, x_{2}^{\prime}\right)$ and $\hat{v}_{0, E}\left(k, x_{2}^{\prime}\right)$, respectively. Using (A.6) we obtain

$$
\frac{\partial^{2} W}{\partial x_{2}^{\prime 2}}-\left(k^{2}+z^{2}\right) W=-\lambda\left(k, x_{2}^{\prime}, z\right),
$$

where

$$
\lambda\left(k, x_{2}^{\prime}, z\right) \equiv G\left(k, x_{2}^{\prime}, z\right)+z \hat{u}_{0, E}\left(k, x_{2}^{\prime}\right)+\hat{v}_{0, E}\left(k, x_{2}^{\prime}\right)
$$


Solving (A.15) gives

$$
\begin{aligned}
W\left(k, x_{2}^{\prime}, z\right)= & \frac{e^{a x_{2}^{\prime}}}{2 a} \int_{x_{2}^{\prime}}^{\infty} e^{-a \xi} \lambda(k, \xi, z) d \xi \\
& +\frac{e^{-a x_{2}^{\prime}}}{2 a} \int_{0}^{x_{2}^{\prime}} e^{a \xi} \lambda(k, \xi, z) d \xi+C(k, z) e^{-a x_{2}^{\prime}},
\end{aligned}
$$

where $C(k, z)$ has to be determined and

$$
a=a(k, z)=\sqrt{k^{2}+z^{2}}
$$

where the square root is chosen as the one having a positive real part for complex numbers away from the negative real axis.

Note that A.7) implies that $W(k, 0, z)$ admits an analytic extension on $k$ in the half-plane $\{\operatorname{Im}(k)<0\}$. On the other hand (A.12) implies that $\frac{\partial W}{\partial x_{2}^{\prime}}(k, 0, z)$ is analytic in $\{\operatorname{Im}(k)>0\}$. Using A.16) it follows that

$$
a(k, z) \psi(k, z)-a(k, z) C(k, z) \text { is analytic in }\{\operatorname{Im}(k)>0\}
$$

and

$$
\psi(k, z)+C(k, z) \text { is analytic in }\{\operatorname{Im}(k)<0\},
$$

where

$$
\psi(k, z) \equiv \int_{0}^{\infty} \frac{e^{-a \xi} \lambda(k, \xi, z)}{2 a} d \xi .
$$

We now introduce the classical Wiener-Hopf decomposition:

$$
a(k, z)=\sqrt{k^{2}+z^{2}}=\sqrt{k+i z} \sqrt{k-i z}=a_{+}(k, z) a_{-}(k, z),
$$

where $\operatorname{Re} z>0$ and then $a_{+}$and $a_{-}$are analytic in the upper and lower half-planes, respectively. For a given function $f \in L^{1}(\mathbb{R})$ we set

$$
\begin{aligned}
& f_{+}(k)=\frac{f(k)}{2}+\frac{1}{2 \pi i} P V \int_{\mathbb{R}} \frac{f(\xi)}{\xi-k} d \xi, \\
& f_{-}(k)=\frac{f(k)}{2}-\frac{1}{2 \pi i} P V \int_{\mathbb{R}} \frac{f(\xi)}{\xi-k} d \xi .
\end{aligned}
$$

We recall that $f_{+}$is analytic in $\{\operatorname{Im}(k)>0\}, f_{-}$is analytic in $\{\operatorname{Im}(k)<0\}$ and that

Let us define

$$
f=f_{+}+f_{-} .
$$

$$
P(k, z) \equiv\left(a_{-} \psi\right)_{+}-\left(a_{-} \psi\right)_{-}+a_{-} C .
$$

Using (A.17), A.18) it follows that

$$
P(k, z) \text { is analytic in }\{\operatorname{Im}(k)>0\}
$$

and

$$
P(k, z) \text { is analytic in }\{\operatorname{Im}(k)<0\} .
$$

The only points where $P(k, z)$ could be singular are $k=0$ and $k=\infty$. Using the asymptotic behaviors of $a_{+}, a_{-}$, as well as A.19) we obtain that $P(k, z)$ is bounded in a neighborhood of the origin and $P(k, z) \rightarrow 0$ as $|k| \rightarrow \infty$. Therefore $P(k, z)$ is globally analytic and by Liouville's theorem $P(k, z)=0$. Then

$$
C(k, z)=\frac{1}{a_{-}}\left[\left(a_{-} \psi\right)_{-}-\left(a_{-} \psi\right)_{+}\right] .
$$


Using this formula as well as (A.16) we can compute $u_{E}\left(x_{1}^{\prime}, x_{2}^{\prime}, t^{\prime}\right)$ by inverting the corresponding Laplace and Fourier transform. However, we are particularly interested in describing the behavior of $u_{E}$ as $\left|\mathbf{x}^{\prime}\right| \rightarrow 0$. To this end we only need to compute the asymptotics of $W\left(k, x_{2}^{\prime}, z\right)$ as $k \rightarrow+\infty, x_{2}^{\prime} \rightarrow 0$. In view of (A.20), (A.21) we obtain

$$
C(k, z) \sim\left[\frac{1}{\pi i} \int_{\mathbb{R}} \sqrt{\xi-i z} \psi(\xi, z) d \xi\right] \frac{1}{k^{3 / 2}} \text { as } k \rightarrow+\infty .
$$

Then using (A.16) as well as the inversion formulae for Laplace and Fourier transforms, we see that

$$
u\left(\mathbf{x}^{\prime}, t^{\prime}\right) \sim \beta\left(t^{\prime}\right) \operatorname{Re}\left(\sqrt{\mathbf{x}^{\prime}}\right) \text { as }\left|\mathbf{x}^{\prime}\right| \rightarrow 0,
$$

where

$$
\beta\left(t^{\prime}\right) \equiv \frac{\sqrt{2} e^{\frac{i \pi}{4}}}{\pi^{2} i} \int_{\tau-i \infty}^{\tau+i \infty} e^{t^{\prime} z}\left[\int_{\mathbb{R}} \sqrt{\xi-i z} \psi(\xi, z) d \xi\right] d z, \quad x^{\prime} \rightarrow 0^{+}, \tau>0 .
$$

Recalling (A.3)- A.5 we obtain a formula similar to (3.34) with

$$
h(t, V t) \equiv-\frac{\sqrt{1-V}}{\left(1-V^{2}\right)^{1 / 4}} \frac{e^{\frac{i \pi}{4}}}{\sqrt{2} \pi^{2} i} \int_{\tau-i \infty}^{\tau+i \infty} e^{\frac{t z}{\sqrt{1-V^{2}}}}\left[\int_{\mathbb{R}} \sqrt{\xi-i z} \psi(\xi, z) d \xi\right] d z .
$$

In the case of Neumann boundary conditions, a similar argument yields

$$
h(t, V t)=\frac{\sqrt{1+V}}{\left(1-V^{2}\right)^{1 / 4}} \frac{e^{-\frac{i \pi}{4}}}{\sqrt{2} \pi^{2} i} \int_{\tau-i \infty}^{\tau+i \infty} e^{\frac{t z}{\sqrt{1-V^{2}}}}\left[\int_{\mathbb{R}} \sqrt{\xi+i z} \psi(\xi, z) d \xi\right] d z
$$

\section{ACKNOWLEDGEMENTS}

This work has been partially supported by DGES Grant BFM2000-0605.

\section{REFERENCES}

[1] M. Amestoy and J.B. Leblond: Crack paths in plane situations - II. Detailed form of the expansion of the stress intensity coefficient. Int. J. Solid Structures 29 (1992), 465-501. MR1138337

[2] J.D. Eshelby: The elastic field of a crack extending nonuniformly under general antiplane loading. Journal of the Mechanics and Physics of Solids 17 (1969), 177-199.

[3] G.A. Francfort and C.J. Larsen: Existence and Convergence for Quasi-Static Evolution in Brittle Fracture, Comm. Pure and Appl. Math. 56 (2003), 1465-1500. MR.1988896 (2004d:49003)

[4] L.B. Freund: Dynamic Fracture Mechanics. Cambridge University Press (1998). MR:1054375 (92f:73044)

[5] A. Friedman, B. Hu and J.J.L. Velázquez: The evolution of stress intensity factors and the propagation of cracks in elastic media. Arch. Rat. Mech. Anal. 152 (2000), 103-139. MR:1760415 (2001h:74026)

[6] A. Friedman, B. Hu and J.J.L. Velázquez: The evolution of stress intensity factors in the propagation of two dimensional cracks. Euro. Jnl. of Appl. Math. 11 (2000), 453-471. MR1799921 (2001h:74027)

[7] J. Herrmann and J.R. Walton: A new method for solving dynamically accelerating crack problems. Part I: The case of a semi-infinite mode III crack in elastic material revisited, Quart. Appl. Math. 50, No. 2 (1992), 373-387. MR1162281 (93c:73086)

[8] M.A. Herrero, G.E. Oleaga and J.J. Velázquez: A note on planar cracks running along piecewise linear paths. Proceedings of the Royal Society of London A 460 (2004), 581-601. MR2034657 (2004j:74116) 
[9] F. John: Partial Differential Equations. Springer-Verlag, New York (1978). MR0514404 (80f:35001)

[10] B.V. Kostrov: On the crack propagation with variable velocity. International Journal of Fracture 11 (1975), 47-56.

[11] J.B. Leblond: Crack paths in plane situations - I. General forms of the expansion of the stress intensity factors. Int. J. Solid Structures 25 (1981), 1311-1325. MR.1138834

[12] T.L. Leise and J.R. Walton, A general method for solving dynamically accelerating multiple co-linear cracks, Int. J. Frac. 111 (2001), 1-16.

[13] B.Noble: Methods based on the Wiener-Hopf Technique. Pergamon, New York (1958).

[14] V. Saraikin and L. Slepyan: Plane problem of the dynamics of a crack in an elastic solid, Mechanics of Solids 14 (1979), 46-62. MR0572877 (81c:73052)

[15] J.R. Willis, Accelerating cracks and related problems, in Elasticity: Mathematical Methods and Applications, Ed. G. Eason and R. W. Ogden, Ellis Horwood, Chichester (1990), 397-409.

Departamento de Matemática Aplicada, Facultad de Matemáticas, Universidad ComPLUTEnse, Madrid 28040, Spain

Departamento de Matemática Aplicada, Facultad de Matemáticas, Universidad ComPlutense, Madrid 28040, Spain

Departamento de Matemática Aplicada, Facultad de Matemáticas, Universidad ComPLUTENSE, MADRID 28040, SPAIN 